Chapman University

Chapman University Digital Commons

Pharmacy Faculty Articles and Research

School of Pharmacy

$5-2004$

\title{
Synthesis of Nanocrystalline Nickel-Zinc Ferrites via a Microemulsion Route
}

Vuk Uskoković

Chapman University, uskokovi@chapman.edu

Miha Drofenik

Jožef Štefan Institute

Follow this and additional works at: http://digitalcommons.chapman.edu/pharmacy_articles

Part of the Nanotechnology Commons, Other Chemistry Commons, and the Physical Chemistry Commons

\section{Recommended Citation}

V. Uskoković, M. Drofenik, "Synthesis of Nanocrystalline Nickel-Zinc Ferrites via a Microemulsion Route", Materials Science Forum, Vols. 453-454, pp. 225-230, 2004

doi: 10.4028/www.scientific.net/MSF.453-454.225

This Article is brought to you for free and open access by the School of Pharmacy at Chapman University Digital Commons. It has been accepted for inclusion in Pharmacy Faculty Articles and Research by an authorized administrator of Chapman University Digital Commons. For more information, please contact laughtin@chapman.edu. 


\section{Synthesis of Nanocrystalline Nickel-Zinc Ferrites via a Microemulsion Route}

\section{Comments}

This is a pre-copy-editing, author-produced PDF of an article accepted for publication in Materials Science Forum, volumes 453-454, in 2004 following peer review. The definitive publisher-authenticated version is available online at DOI: 10.4028/www.scientific.net/MSF.453-454.225.

\section{Copyright}

Trans Tech Publications 


\title{
Synthesis of Nanocrystalline Nickel-Zinc Ferrites via a Microemulsion Route
}

\author{
V. Uskoković, M. Drofenik ${ }^{* *}$ \\ * »Jožef Stefan « Institute, Jamova 39, 1000 Ljubljana \\ ${ }^{* *}$ Faculty of Chemistry and Chemical Engineering, Smetanova 17, 2000 Maribor
}

Keywords: Reverse micelle, Ferrite, Nanomaterial, Co-precipitation, CTAB, pH

\begin{abstract}
Nanostructured NiZn-ferrites were synthesized using a low-temperature co-precipitation method within the reverse micelles of ternary CTAB/1-hexanol/water microemulsions. The influence of $\mathrm{pH}$ on the precipitation reaction was investigated. It was found that a higher $\mathrm{pH}$ resulted in more crystalline particles. Subsequent oxidation of the ferrous ions precipitate, which leads to the formation of spinel ferrites, is discussed. Diffraction-lines broadening and specific surface area measurements yielded an average particle size of $\sim 3 \mathrm{~nm}$. The saturation magnetization of the samples was 20 times smaller than for traditionally synthesized NiZn-ferrites, while the coercivities were 10 - 100 times larger than bulk NiZn-ferrites, which is consistent with the nanocrystalline nature of the synthesized magnetic material. TEM investigations were used to reveal the agglomerated nature of the synthesized samples, which is the reason why the samples do not show superparamagnetic behaviour.
\end{abstract}

\section{Introduction}

Nickel-zinc ferrites [1] are of great interest to modern electronic industry since they have been used as many electronic components [2] such as transformer cores, giant magneto-resistant materials, recording heads [3], antennas or loading coils. The main properties of NiZn-ferrites, which make them unique magnetic ceramic material are their high electrical resistivities, which in turn lead to lower eddy current losses, the losses that become significant at EM fields of high frequencies. The main idea behind the production of nanostructured NiZn-ferrites is that their electrical resistivity might be pushed to still higher values due to increased concentration of grain boundaries [4]. Deagglomerated nanosized and single-domain magnetic particles are constituent parts of magnetic fluids [5], which are used in novel dynamic speakers, acoustic devices [6] and "magnetic vs. non-magnetic" separators.

Different low-temperature routes (often called wet routes) for synthesis of ferrite powders, such as co-precipitation [7], sol-gel [8], hydrothermal syntheses [9] and others are described in the literature. All of these low-temperature methods were primarily invented in order to overcome difficulties arising out of the traditional high-temperature way of ferrites syntheses, which feature weak control over the final composition, chemical inhomogeneity, broad distribution of particle sizes, and inevitable product's contamination with impurities during the stages of grinding and mixing. It is known that by using a relatively novel (21 years old [10]) reverse-micellemicroemulsion method of synthesis, particles of controllable and uniform sizes and shapes might be obtained [11]. Relatively low processing costs and low syntheses temperatures make the microemulsion-assisted synthesis of ferrite particles alluring for various applications. Multiple microemulsion approaches, where different reactants are closed inside the respective reverse 
micelles, are within previously mentioned class proven to be more successful route [12] comparing to single-microemulsion approach where the reactants are let to diffuse in water cores of reverse micelle where the chemical reactions of the synthesis take place.

\section{Experimental}

The ternary microemulsion CTAB/water/1-hexanol was used as the medium in which $\mathrm{Ni}_{0.5} \mathrm{Zn}_{0.5} \mathrm{Fe}_{2} \mathrm{O}_{4}$ was synthesized. The following chemicals were used: $\mathrm{FeSO}_{4} \cdot 7 \mathrm{H}_{2} \mathrm{O}(>99 \%$, Alfa Aesar), $\mathrm{NiSO}_{4} \cdot 7 \mathrm{H}_{2} \mathrm{O}$ (99,7\%, Podnart), $\mathrm{ZnSO}_{4} \cdot 7 \mathrm{H}_{2} \mathrm{O}$ (>99\%, Alfa Aesar) as precursor salts; CTAB (>99\%, Alfa Aesar) as the surfactant; 1-hexanol (>98\%, Merck-Schuchardt) as the oil phase; deionized water as the aqueous phase; $\left(\mathrm{CH}_{3}\right)_{4} \mathrm{NOH}(25 \mathrm{wt} \%, 99,5 \%$ purity, Alfa Aesar) as the precipitating agent; $\mathrm{H}_{2} \mathrm{O}_{2}$ (30 wt \%, Carlo Erba) as the oxidating agent; $\mathrm{H}_{2} \mathrm{SO}_{4}$ (99,8 \%, Carlo Erba) as the acidifying agent; ethanol (99,8 \%, Carlo Erba) and deionized water as the washing substances. The chemical composition (CTAB:hexanol: $\mathrm{H}_{2} \mathrm{O}=30.5: 57: 12.5$ ) of the templating reverse micellar microemulsion for in situ synthesis of NiZn-ferrite was chosen from the previous work [13]. When using a divalent iron salt as precursor component, $\mathrm{pH}$ of the solution must be under 1,8 so as to avoid early oxidation of ferrous ions.

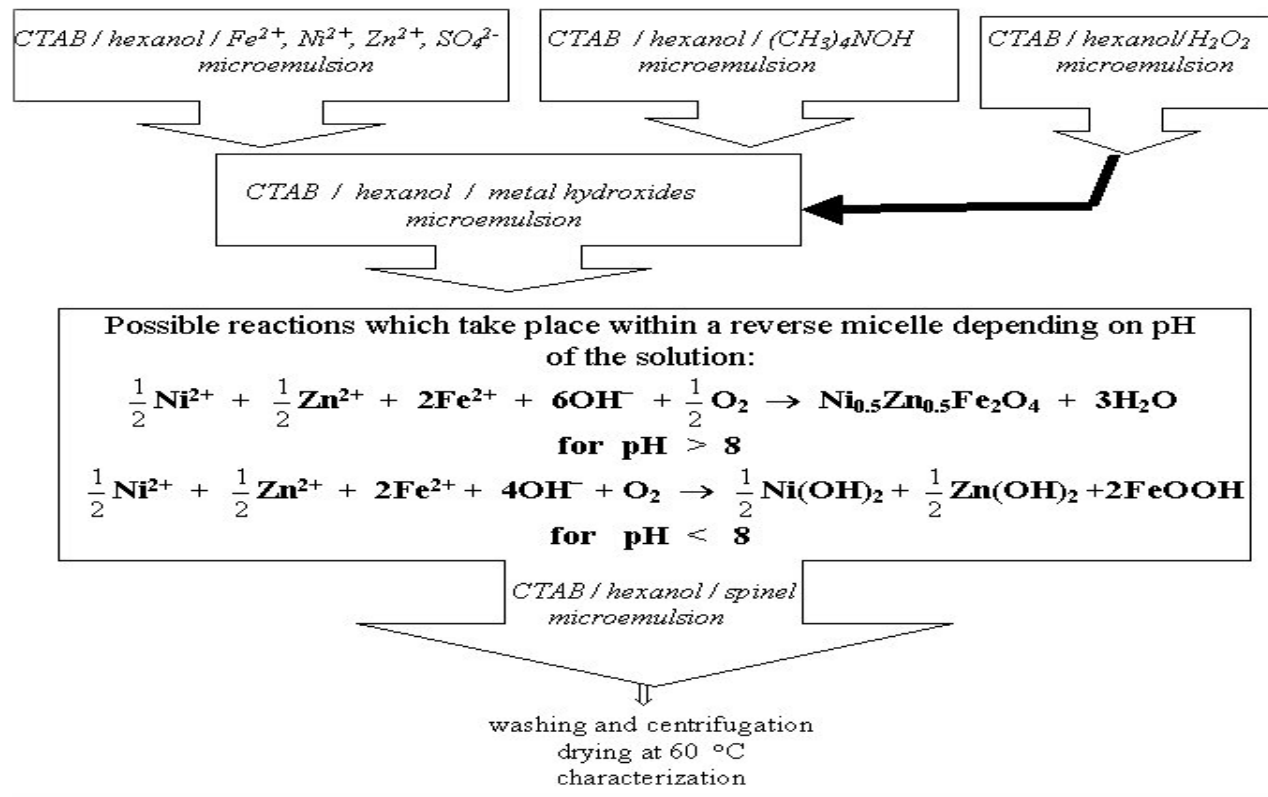

Fig.1. Flow chart of the NiZn-ferrite nano-powder synthesizing procedure by using the microemulsion route.

The formation of NiZn-ferrite particles by hydrolyzing and subsequent oxidizing the alkaline suspension of hydroxides $\mathrm{Fe}(\mathrm{OH})_{2}, \mathrm{Ni}(\mathrm{OH})_{2}$ and $\mathrm{Zn}(\mathrm{OH})_{2}$ is based on the Schikorr's reaction [14]. The reaction proceeds within the "water pools" of reverse micelles and is governed by oxidation of the mixture of metal hydroxides of an appropriate composition in an alkaline media. The synthesis route of the produced NiZn-ferrites is shown in Fig.1. Three separate microemulsions under controlled temperature on air were applied for obtaining the nanosized NiZn-ferrite. The first one comprised aqueous solution of precursor cations in total concentration of $0.237 \mathrm{M}$ and with $\mathrm{pH}=1.6$, the second microemulsion comprised aqueous $5 \mathrm{wt} \%\left(\mathrm{CH}_{3}\right)_{4} \mathrm{NOH}$ aqueous solution, while the third microemulsion carried $30 \mathrm{wt} \% \mathrm{H}_{2} \mathrm{O}_{2}$ aqueous solution in its "water pools". 
Obtained nanoparticles' morphologies were investigated by using TEM (JEOL JEM2000FX). The powders were also characterized by using the X-ray diffraction analyses (D4 Endeavour), specific magnetization measurements (MANICS DSM10) and specific surface-area measurements (Micromeritics Gemini II analyzer).

\section{Results and discussion}

The X-ray diffraction patterns of the synthesized nano-powders at the precipitating $\mathrm{pH}$ values higher than 8 and below 8 are shown in Fig.2.

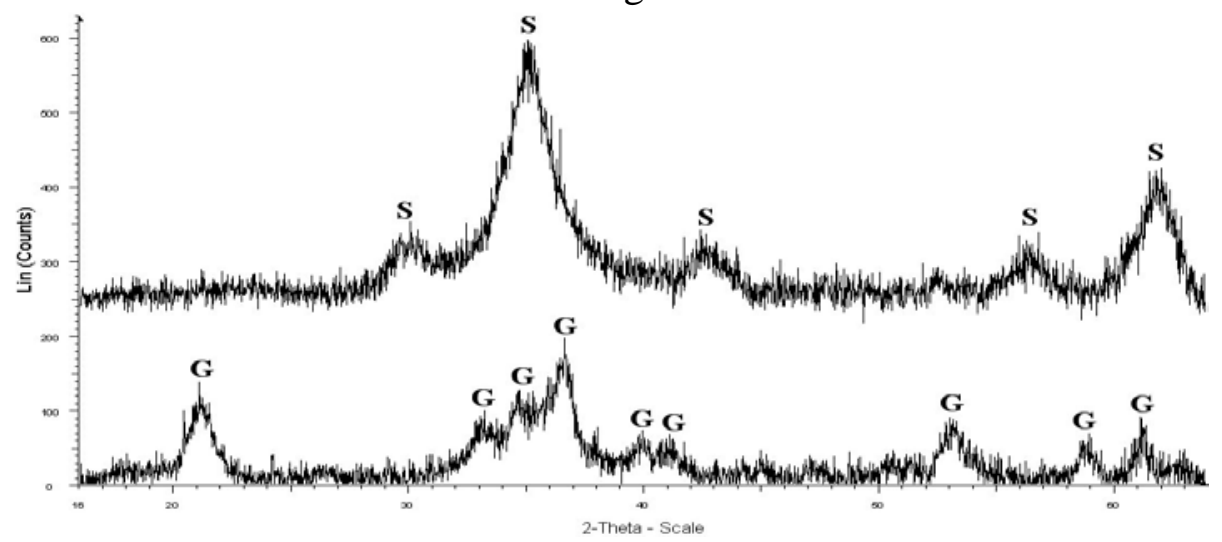

Fig. 2. X-ray patterns of the sample synthesized at the $\mathrm{pH}$ of the precipitation below 8 (down) and above 8 (up). Spinel-derived peaks are denoted with S, while goethite-derived peaks are denoted with G.

During the oxidation of the microemulsion two concurrent reactions depending on the hydroxide ion concentration take place. The first reaction is associated with the oxidation of the iron(II) hydroxide and incorporation of $\mathrm{Fe}^{3+}$ into spinel NiZn-ferrite, while the second reaction leads to the formation of goethite $(\alpha-\mathrm{FeOOH})$, which might also persist as an intermediate compound in the formation of ferrite.

Simplified chemical reactions for the both processes can be written as:

$$
\begin{aligned}
& 1 / 2 \mathrm{Ni}^{2+}+1 / 2 \mathrm{Zn}^{2+}+2 \mathrm{Fe}^{2+}+6 \mathrm{OH}^{-}+1 / 2 \mathrm{O}_{2} \rightarrow \mathrm{Ni}_{0.5} \mathrm{Zn}_{0.5} \mathrm{Fe}_{2} \mathrm{O}_{4}+3 \mathrm{H}_{2} \mathrm{O} \\
& 2 \mathrm{Fe}^{2+}+4 \mathrm{OH}^{-}+1 / 2 \mathrm{O}_{2} \rightarrow 2 \mathrm{FeOOH}+\mathrm{H}_{2} \mathrm{O}
\end{aligned}
$$

At sufficiently high concentrations of hydroxyl ions, i.e. at $\mathrm{pH}>8$, NiZn-ferrite is formed, while at lower $\mathrm{pH}$ values, goethite appears as the main phase. In order to achieve a higher $\mathrm{pH}$ value when following a multimicroemulsion approach, the amount of the alkaline microemulsion was varied so that the parameter $\mathrm{w}$, that is molar ratio of water to surfactant, remains the same during the course of every experiment. This can be observed in the Table I where the particle size is of the same order of magnitude for all the samples, but slightly larger for the samples synthesized at higher $\mathrm{pH}$ values (11-13.5). It is well-known that the main factor for regulating the average particle size of the synthesized samples is parameter w, although dynamic intermicellar interaction is more recently paid attention to, as well. The calculated diameter of the water pools [15] for the given weight percentage of water $(12.5 \%)$ is equal to $2.4 \mathrm{~nm}$, which is very close to the average particle sizes estimated from the broadening of diffraction lines $(2-4 \mathrm{~nm})$. 
Table II. Sample ID, $\mathrm{pH}$ of the precipitation, identified crystalline phases, specific surface area $\left(A_{s}\right)$, average grain size obtained from the measured specific surface $\left(d_{s}\right)$ and $x$-ray broadening measurements $\left(d_{x}\right)$, and saturation magnetization $\left(M_{s}\right)$

\begin{tabular}{|c|c|c|c|c|c|c|}
\hline Sample ID & $\mathrm{pH}$ & Identified phases & $\begin{array}{c}\mathrm{A}_{\mathrm{s}} \\
\left(\mathrm{m}^{2} / \mathrm{g}\right)\end{array}$ & $\begin{array}{c}\mathrm{d}_{\mathrm{s}} \\
(\mathrm{nm})\end{array}$ & $\begin{array}{c}\mathrm{d}_{\mathrm{x}} \\
(\mathrm{nm})\end{array}$ & $\begin{array}{c}\mathrm{M}_{\mathrm{s}} \\
(\mathrm{emu} / \mathrm{g})\end{array}$ \\
\hline $\mathrm{F}$ & 4 & Goethite & - & - & - & 0.8 \\
\hline $\mathrm{G}$ & 4 & Goethite & - & - & - & 0.8 \\
\hline $\mathrm{H}$ & 8 & Spinel & 212 & 6 & 2 & 1.2 \\
\hline $\mathrm{I}$ & 9 & Spinel & 305 & 4 & 3 & 0.9 \\
\hline $\mathrm{J}$ & 10 & Spinel & 318 & 4 & 2 & 1.1 \\
\hline $\mathrm{K}$ & 11 & Spinel & 303 & 4 & 4 & 4.2 \\
\hline $\mathrm{L}$ & 12 & Spinel & 261 & 5 & 4 & 4.6 \\
\hline $\mathrm{M}$ & 13 & Spinel & 350 & 4 & 4 & 4.6 \\
\hline $\mathrm{N}$ & 13,5 & Spinel & 333 & 4 & 4 & 5.4 \\
\hline
\end{tabular}

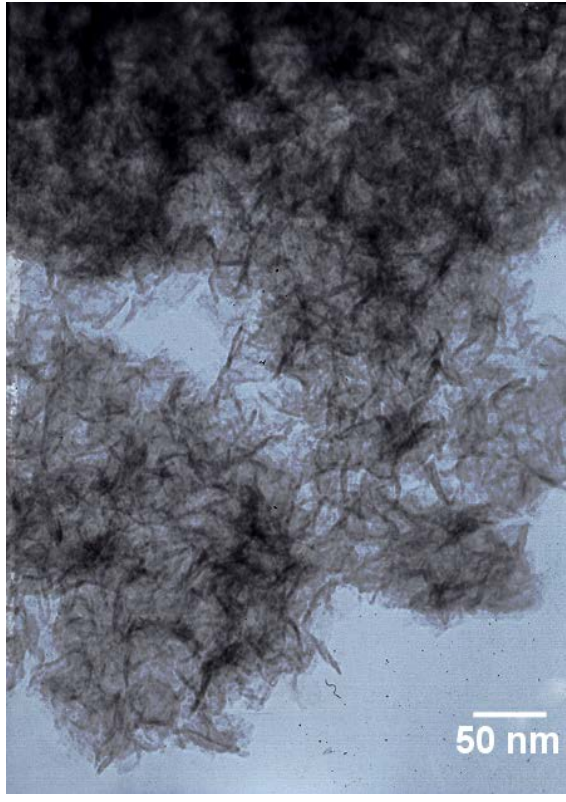

a.)

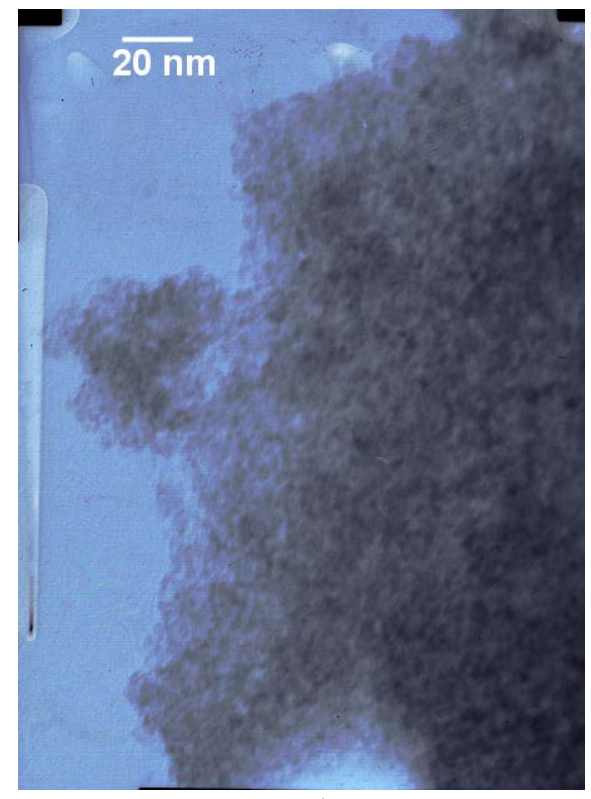

b.)

Fig. 3. TEM images of the sample I (a) and the sample K (b).

TEM images of the powders I and $\mathrm{K}$ are shown in Fig.3. The sample I, just as all the synthesized samples between the precipitating $\mathrm{pH}$ values of 8 and 10, is featured by acicular particles, and consequently, due to their high anisotropy, large values of coercivities. On the other hand, the samples synthesized at the precipitating values $\geq 11$ consist of uniform-sized agglomerated particles. The agglomerated nature of the synthesized powders is the reason for they do not obey superparamagnetic model [16]. The measured coercivities varied from 24 Oe to 1300 Oe, which is in the latter case about 100 of times larger comparing to bulk samples of the same chemical composition. Acicular particles in the samples synthesized at medium $\mathrm{pH}$ values might be derived from the acicular nature of a certain FeOOH phase, which is probably an intermediate ferric compound during the process of formation of NiZn-ferrite. Otherwise, medium $\mathrm{pH}$ values might 
promote spherical-to-worm-like transition of CTAB reverse micelles, which might then serve as templates for the synthesis of acicular crystallites [17].

A typical hysteresis loop of a synthesized NiZn-ferrite nano-powder via the microemulsion route is shown in Fig. 4a, whereby the dependence of saturation magnetization of the samples upon the $\mathrm{pH}$ value of the precipitation is shown in Fig. 4b.

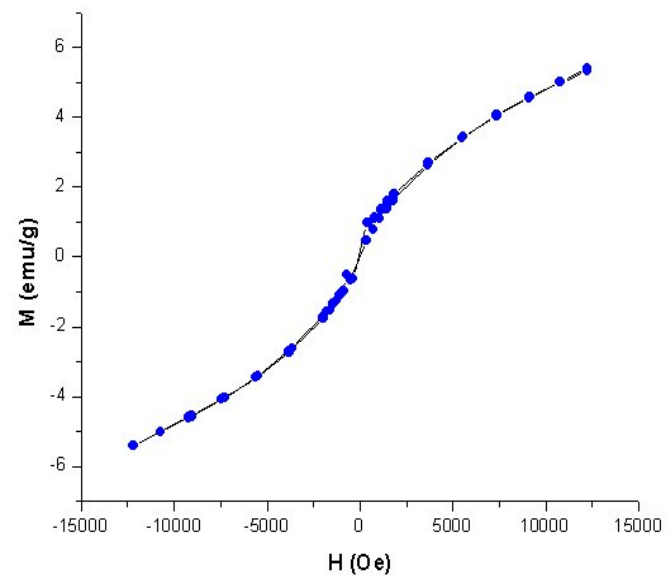

a.)

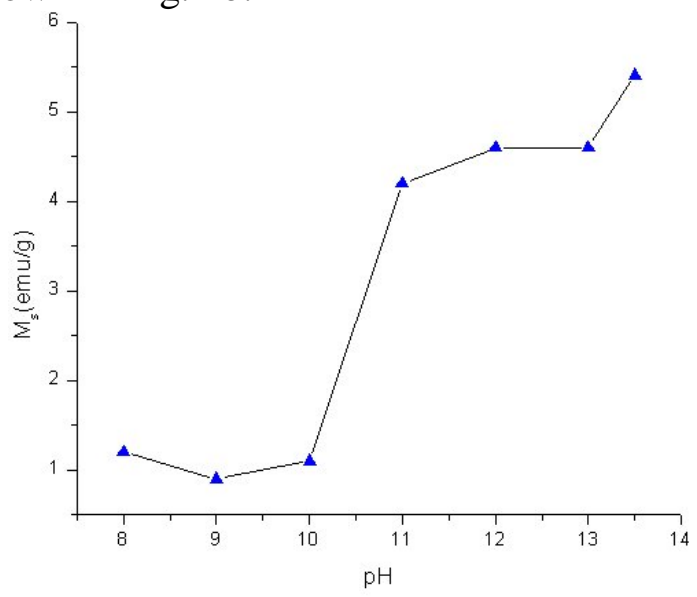

b.)

Fig.4a,b. a.) The hysteresis loop of the nano-sized NiZn-ferrite powder N;b.) Saturation magnetization of the samples versus $\mathrm{pH}$ of the precipitation

The saturation magnetizations of the samples have much smaller values comparing to the values characteristic for bulk-synthesized samples. It is well established that the size of magnetic particles has an influence on the resulting saturation magnetization of the material $[18,19]$. This phenomenon was observed in several ferrite systems [20] and was explained by a magnetically "dead" layer on the surface of particles [21], which is the consequence of non-collinear surface spins. Many other reasons such as crystal lattice defects, the mass effects of absorbed water, chemical and physical changes on the surface, magnetic degradation of the surface or redistribution of cation sites in the lattice might be taken into account when considering decrease in magnetization with reducing the average particle size. Since the average particle sizes of the synthesized NiZnferrites are found to be in range of 2-4 nm, about half of all the atoms in the material are positioned on the particles' surfaces. An increase of the size of crystallite domains as determined from XRDlines-broadening measurements, between precipitating $\mathrm{pH}$ values of 10 and 11 (Table 1) is reflected on a 4-fold increase in saturation magnetization between these two synthesizing pH values (Fig. 4b). Nevertheless, even at the precipitating $\mathrm{pH} \geq 11$, the samples' magnetizations are smaller by a factor of about 20 comparing to bulk-synthesized NiZn-ferrites, and this ratio of magnetizations for NiZnferrites synthesized using traditional and microemulsion routes was already predicted in the literature [22].

Prolonging or shortening the time between the initiated precipitating reaction and the isolation of magnetic particles (which was usually 1 hour) did not leave any significant traces on magnetic or crystalline characteristics of the powder. It was only revealed that the coercivity of the samples decreases with the aging time (from 1300 Oe to 800 Oe to 400 Oe as the aging time was prolonged from $30 \mathrm{~min}$ to $1 \mathrm{~h}$ to $2 \mathrm{~h}$ for the sample $\mathrm{H}$ ), which might be related to the refinement of the surface of the particles, so that they can more easily change the direction of magnetic moments.

The purpose of the third, oxidating microemulsion is still not clear since there were no changes in crystallinity at $\mathrm{H}_{2} \mathrm{O}_{2}$-oxidized and $\mathrm{H}_{2} \mathrm{O}_{2}$-non-oxidized samples, whereas the $\mathrm{H}_{2} \mathrm{O}_{2}$ - 
oxidized sample had for about $50 \%$ higher magnetization than the $\mathrm{H}_{2} \mathrm{O}_{2}$-non-oxidized sample. Thus, completion of the dissolved oxygen or the decomposition of water-initiated [23] oxidation of ferrous ions, which along the way serve as spinel crystallization nuclei and refinement of spinel particles initiated by exothermic reaction of oxidation might be taken into account to happen when using this step in procedure.

\section{Conclusions}

The results presented here show that the performed low-temperature co-precipitation technique within the reverse micelles of the microemulsion system CTAB/1-hexanol $/ \mathrm{H}_{2} \mathrm{O}$ by using triple-microemulsion procedure is appropriate for the synthesis of nanostructured NiZn-ferrite particles of uniform sizes. $\mathrm{pH}$ value of the precipitation reaction should exceed 8 in order to yield spinel as the main phase, and should exceed 11 in order to gain particles of uniform morphology. With the increase in precipitating $\mathrm{pH}$, the magnetization increases too, while the XRD-determined average particle size remains at $\sim 4 \mathrm{~nm}$. TEM investigations revealed coral-like agglomerated nature of the nanoparticles synthesized at higher $\mathrm{pH}$ value, while the existence of acicular particles was observed within the sample synthesized at medium $\mathrm{pH}$ values. Increase in aging time leads to the decrease in large coercivities, which suggests refinement of particles in microemulsion in time.

\section{References}

1. A. Goldman, Modern Ferrite Technology, Van Nostrand Reinhold (1990).

2. T. Abraham, Am. Cer. Soc. Bull. 73, 62 (1994).

3. J. A. Burg, T. C. Anthony, J. H. Nickel, Mater. Res. Bull. 21(9) 23-27(1996).

4. C. R. Hendricks, V. W. R. Amarakoon, D. Sullivan, Cer. Bull. 70 (5) 817 - 823 (1991).

5. K. Raj, B. Moskowitz, R. Casciari, J. Magn. Magn. Mater. 149, 174 - 180 (1995).

6. V. E. Fertman, Magnetic Fluids Guidebook, Hemisphere Publ. Corp., New York (1990).

7. Soft Ferrites, A Users's Guide; Magn. Mater. Producers Association, Chicago, IL, (1997).

8. A. Goldman and A.M. Laing, J. Phys.(Paris) 4 (38) C1-297-C1-301 (1977).

9. M. Rozman and M. Drofenik, J. Am. Ceram. Soc. 78 (9) 2449-55 (1995).

10. M. Boutonnet, J. Kizling, P. Stenius, Coll. \& Surf. 5 (3), 209 - 225 (1982).

11. D. O. Yener, H. Giesche, J. Am. Cer. Soc. 84 (9) 1987-95 (2001).

12. J. Wang, P. F. Chong, S. C. Ng, L. M. Gan, Mater. Lett. 30, 217 - 221 (1997).

13. V. Uskoković, M. Drofenik, Mater. \& Tech. 37 (3-4) 129 - 131 (2003).

14. G. Schicorr, Z. Allgem. Chem. 212, 33-38 (1938).

15. E. Rodenas, M. Valiente, Coll. \& Surf. 62, 289 - 295 (1992).

16. C. P. Bean, J. D. Livingston, J. Appl. Phys. 30 (4) 120S - 129S (1959).

17. S. Xu, H. Zhou, J. Xu, Y. Li, Langmuir 18, 10503 - 10504 (2002).

18. R. H. Kodama, J. Magn. Magn. Mater. 200, 359 - 372 (1999).

19. J. P. Chen, C. M. Sorenson et al, Phys. Rev. 54 (13), 9288-96 (1996).

20. T. Sato, T. Iijima, M. Seki, N. Inagaki, J. Magn. Magn. Mater. 65, 252 (1987).

21. A. E. Berkowitz, W. J. Schule, P. J. Flanders, J. Appl. Phys. 39, 1261 (1987).

22. S. Santra, R. Tapec, N. Theodoropoulou et al., Langmuir 17, 2900 - 2906 (2001).

23. H. Robbins, Proceedings of the ICF, Center for Academic Publications, Japan (1981).

24. Y. Todaka, M. Nakamura, S. Hattori et al., Mater. Trans. 44 (2) 277 - 284 (2003). 
